Portland State University

PDXScholar

$11-16-2018$

\title{
NORSE and Super-Refractory Status Epilepticus: a Single-Center Retrospective Chart Review
}

Manaz Rezayee

Portland State University

Follow this and additional works at: https://pdxscholar.library.pdx.edu/honorstheses

Let us know how access to this document benefits you.

\section{Recommended Citation}

Rezayee, Manaz, "NORSE and Super-Refractory Status Epilepticus: a Single-Center Retrospective Chart Review" (2018). University Honors Theses. Paper 675.

https://doi.org/10.15760/honors.689

This Thesis is brought to you for free and open access. It has been accepted for inclusion in University Honors Theses by an authorized administrator of PDXScholar. Please contact us if we can make this document more accessible: pdxscholar@pdx.edu. 
NORSE and Super-Refractory Status Epilepticus: A Single-Center Retrospective Chart Review

\author{
by \\ Manaz Rezayee
}
An undergraduate honors thesis submitted in partial fulfillment of the requirements for the degree of
Bachelor of Science
in
University Honors
and
Biology

Thesis Advisor:

Marissa Kellogg M.D., M.P.H

Portland State University 


\section{Acknowledgements}

First, I would like to thank my thesis advisor, Dr. Marissa Kellogg, for being an amazing mentor and for being extremely understanding of the ongoing unexpected difficulties and obstacles. I cannot thank you enough for allowing me, an undergraduate student, to work alongside you in such an advanced and rigorous field, while also constantly encouraging and providing me with more opportunities for exposure in this field. Thank you to Dr. Madeline Nguyen who has worked alongside me throughout the project to analyze the data and allowed me to increase my exposure in Neuro ICU. Also, a big thank you to Chad Murchison for being a great statistician throughout this study. 


\begin{abstract}
Rationale: New-Onset Refractory Status Epilepticus (NORSE) is a rare life-threatening condition in previously healthy patients who develop new-onset medically-refractory status epilepticus of unclear etiology. ${ }^{1}$ NORSE commonly leads to super-refractory status epilepticus (SRSE), a severe form of status epilepticus for which seizures persist despite 24 hours of adequate anti-seizure and anesthetic treatment. The goal of this study is to examine the clinical characteristics and epidemiology of super-refractory NORSE, a subset of SRSE, to determine risk and prognostic factors associated with this rare condition and mortality rates.
\end{abstract}

Methods: We performed a retrospective cohort study of all cases of SRSE admitted to Oregon Health \& Science University, an academic tertiary care center between 2007 and 2016.

Results: Among 358 patients monitored on continuous EEG for at least 4 days in a single admission, 67 (19\%) were identified as having SRSE. In-hospital mortality for SRSE (all causes) was 30\% (20), but for the specific NORSE group 15\% (2), for pre-existing epilepsy $40 \%$ (4) and for symptomatic $32 \%$ (14).

Conclusions: The results of this study are consistent with prior reports of NORSE epidemiology: the condition is rare, patients tend to be younger, female-predominate, and there have been no antibodies clearly-associated with the condition. A novel finding in this study was that $50 \%$ of NORSE cases had positive antibody testing- the majority of which were thyroid-associated antibodies (41.7\%). Based on a population-based study at baseline of 5783 participants, $12.8 \%$ were positive for TPO antibodies with a higher prevalence in women compared to men. ${ }^{2}$ While thyroid-associated antibodies are thought to be non-specific markers of inflammation and/or autoimmunity, this supports the hypothesis that NORSE (or a subset of NORSE cases) may be autoimmune-mediated and establishes a potential autoimmune link.

Keywords: New-Onset Refractory Status Epilepticus, NORSE, Super-Refractory Status Epilepticus, SRSE, Status Epilepticus, Refractory Status Epilepticus, RSE, Seizures, Epilepsy 


\section{Introduction}

Status epilepticus (SE) is a neurological emergency characterized by repetitive seizures without full recovery to neurological baseline between the seizures. ${ }^{3}$ In approximately $23 \%-43 \%$ of SE patients, SE persists despite administration of at least 2 anti-epileptic medications including a benzodiazepine. This condition is known as refractory SE (RSE). ${ }^{4,5} \mathrm{New}-\mathrm{Onset}$ Refractory Status Epilepticus (NORSE) is a rare life-threatening condition in previously healthy patients who develop new-onset RSE of unclear etiology, which was first described in $2005 .{ }^{1} \mathrm{~A}$ critical distinction is that NORSE refers to a clinical presentation or syndrome, and not a specific diagnosis. ${ }^{6}$ NORSE commonly leads to super-refractory status epilepticus (SRSE), a severe form of status for which seizures persist despite 24 hours of adequate anti-seizure and anesthetic treatment.

As a rare recently-described syndrome, the scientific literature on NORSE consists mostly of cases describing the clinical characteristics, demographics and prognosis of small groups of subjects, the largest of which consisted of 130 patients from 13 different centers. Demographic factors associated with NORSE in prior studies includes female gender prevalence, age of onset (more likely at a younger age), ${ }^{1}$ previously good health, increased EEG duration, prolonged hospital stay, longer duration of refractory seizure occurrence and excessive negative work-up.

Typical work-up for the patients has included CT scan, continuous EEG, CSF analysis, and a crucial auto-immune work-up of antibodies (paraneoplastic/autoimmune panel testing, thyroid, or other). Based on previous literature, a CSF work-up is recommended immediately after the admission of the patient as the CSF fluid is a strong indicator for abnormalities in the Central Nervous System and useful to understand the initial symptoms of presentation. ${ }^{7}$ 
Throughout this work-up, several different treatments would be utilized as well such as antiepileptic medication and immunotherapies. Prognosis is guarded; rates of mortality in NORSE range from 20 to $50 \%$ and disability due to NORSE is common. ${ }^{5,6,8,9}$

Due to the refractory nature of NORSE and SRSE, there have been several different treatments that have been used or recommended. Initially, anti-epileptic medications (clonazepam, phenobarbital, etc) and steroids, such as dexamethasone, methylprednisolone and prednisolone, are taken into consideration due to beneficial outcomes reported in previous literature for SRSE patients. ${ }^{10-12}$ Second-line therapy options taken into consideration include immunotherapy (IVIG, PLEX, cyclophosphamide and rituximab). In fact, it is recommended that immunotherapies should be initiated within 72 hours of initial admission. ${ }^{7}$ Although lab results may be prolonged, it is recommended that the planning of early immunotherapy would be beneficial, especially if the results of autoimmune antibody tests are positive. ${ }^{13}$

Third-line of therapy would be Electroconvulsive Therapy (ECT) or the ketogenic diet. ECT is an electrical stimulation of the brain that is administered when the patient is under a slight amount of anesthesia. ${ }^{14}$ Although there are few reports of effectiveness of ECT with patients who had withdrawal or reduction of anesthesia in order to allow ECT to be administered, there is a possibility that the reduction itself resulted to the resolution of SE, and not the ECT. ${ }^{14}$ However, it is important to consider ECT as a third-line treatment as it has been reported to work on a few patients. The ketogenic diet was also suggested as a third-line of therapy with several reports showcasing beneficial results. In one specific study by Kramer et al, a patient was reported suffering from over 100 seizures a day; however, within 2 days of starting the ketogenic diet, the patient had a total cessation of seizures. ${ }^{15}$ Therefore, a ketogenic diet has been reported in several cases showcasing the effectiveness in regards to seizures relating to SE, NORSE and 
FIRES. ${ }^{16}$ FIRES is also a subcategory of NORSE requiring a prior febrile infection with fever prior to the onset of RSE. ${ }^{6}$

The etiology of NORSE remains unclear. While the scientific literature to date hypothesizes that NORSE is an inflammatory autoimmune and/or post-infectious condition, no antibodies have been clearly associated with NORSE to date. ${ }^{8}$ Antibodies typically associated with NORSE are anti-NMDA receptor and anti-VGKC complex (voltage-gated potassium channel), although there are several cases with non-thyroid related (neuronal) antibodies as well. ${ }^{8}$ Our study suggests NORSE may be autoimmune-mediated, possibly thyroid related. Additionally, prognostic factors for survival and typical clinical outcomes have not yet been well-established. The goal of this study is to examine the clinical characteristics and outcomes of all SRSE cases admitted to a single level IV epilepsy center over a 10 -year period, to determine how NORSE cases compare to cases of SRSE of known etiology in terms of epidemiology, workup, treatment and prognosis. 


\section{Methods}

We performed an IRB-approved retrospective chart review of all cases of SRSE admitted to Oregon Health \& Science University (OHSU), a 556-bed academic tertiary care hospital, between 2007 and 2016. ${ }^{17}$ The inclusion criteria for SRSE patients were: 1) continuous EEG monitoring for a minimum of 4 days,

2) EEG-confirmed seizures, and 3) presence of medically-induced burst suppression on EEG. As shown

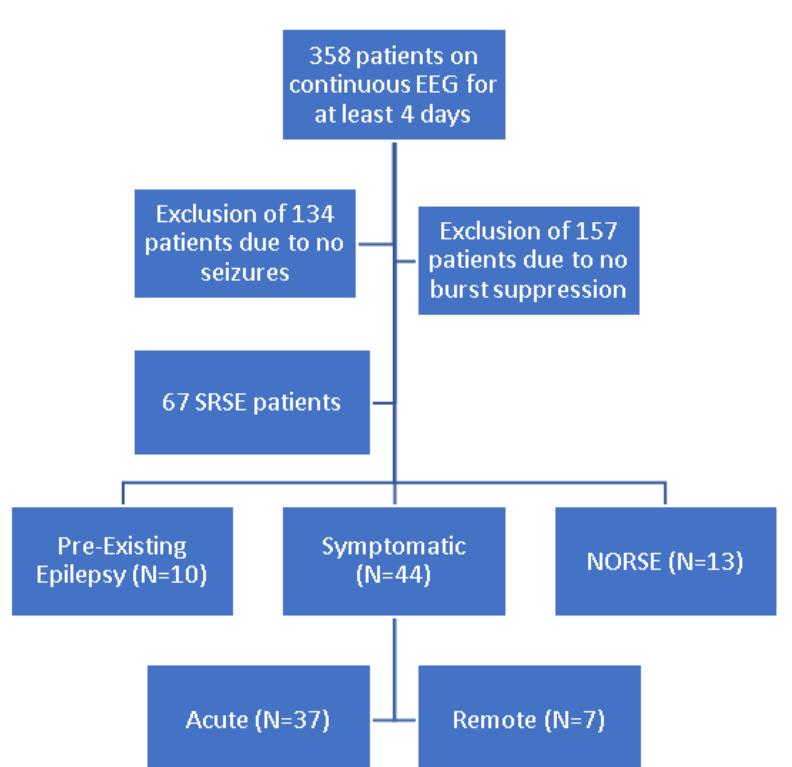
in Figure 1, SRSE patients were further assessed by

Figure 1: Screening of patients for inclusion in this study. determining the etiology, which were categorized by the three dominant conditions: longstanding epilepsy, symptomatic, or NORSE. Symptomatic seizures are known as provoked seizures occurring at the time of or in close temporal association with an evident brain insult such as an infection, traumatic brain injury, electrolyte abnormality or a stroke. ${ }^{18}$ Since NORSE is defined as unknown etiology of RSE, the inclusion criteria to distinguish NORSE patients from other etiologies were: 4) no pre-existing diagnosis of epilepsy and 5) no symptomatic cause of seizures identified within 48 hours of admission.

Due to the severity NORSE, many patients had 2 or more admissions characterized by seizures and burst suppression. In these cases, the admission characterized by more prolonged EEG duration with more numerous seizures was included in the analysis, while the admissions of shorter duration and fewer seizures were excluded. After the SRSE patients are identified, medical charts were examined for the following clinical data: gender, age at onset, duration of EEG, length of stay at OHSU, duration to seize the seizures, if transferred from an outside 
hospital, the date of admission at OHSU, in-hospital mortality, and patients' disposition. NORSE patients were further examined for data such as antibody status (paraneoplastic/autoimmune panel testing, thyroid, or other), CSF abnormality, brain MRI findings, steroid usage, immunotherapies (IVIG, PLEX, cyclophosphamide, and rituximab), fever on initial presentation, number of AEDs at discharge, ECT, ketogenic diet, the modified Rankin Scale (mRS) and autoimmune work-up (antibody testing). A Fisher's Exact test for mortality and gender between NORSE and longstanding epilepsy was completed. Outcomes were assessed by the number of AEDs and Modified Rankin Scale (mRS) at discharge. 


\section{Results}

1. Comparisons of hospital outcomes and mortality between different causes of SRSE

Among 358 patients monitored on continuous EEG for at least 4 days in a single admission, a total of 291 patients were excluded due to no seizures or no incidence of medically induced burst suppression. The remaining 67 patients (19\%) were identified as SRSE. As shown in Figure 1, of the 67 SRSE patients, 10 (15\%) had longstanding epilepsy, 44 (66\%) had symptomatic and $13(19 \%)$ had NORSE. Patients with a symptomatic etiology were assessed further by medical professionals to determine if the patients were acute or remote symptomatic.

Characteristics of SRSE patients collected are shown on Table 1. The median age of onset for both pre-existing epilepsy and symptomatic was 62.5, whereas for NORSE it was 44 years.

Female predominance was shown in symptomatic and NORSE groups by $54.5 \%$ and $69 \%$, respectively. Preexisting epilepsy group was $40 \%$ female gender; therefore, it was not female gender dominant. The median length (days) on the EEG was 12 for epilepsy, 6.5 for symptomatic and 25 for NORSE.
Table 1: Characteristics of the three categorized etiologies (pre-existing epilepsy, symptomatic and NORSE).

\begin{tabular}{|c|c|c|c|}
\hline & $\begin{array}{c}\text { Pre-Existing } \\
\text { Epilepsy (N=10) }\end{array}$ & $\begin{array}{c}\text { Symptomatic } \\
(\mathbf{N}=44)\end{array}$ & $\begin{array}{c}\text { NORSE } \\
(\mathbf{N}=13)\end{array}$ \\
\hline Median Age [IQR] & $62.5[31.5-79]$ & $62.5[50.25-67.75]$ & $44[33-58]$ \\
\hline Female Gender & $40 \%$ & $54.5 \%$ & $69 \%$ \\
\hline $\begin{array}{c}\text { Median length of } \\
\text { EEG [IQR] }\end{array}$ & $12[9-16]$ & $6.5[5-10]$ & $25[12.5-56]$ \\
\hline $\begin{array}{c}\text { Median time to last } \\
\text { seizure [IQR] }\end{array}$ & $5[1.75-8.25]$ & $5[2-9.75]$ & $10[4.5-37.5]$ \\
\hline \begin{tabular}{c} 
In-hospital Mortality \\
\hline Median length of
\end{tabular} & $40 \%(4)$ & $32 \%(14)$ & $15 \%(2)$ \\
\hline stay at OHSU [IQR] & $14.5[11.5-25]$ & $19.5[10-35.75]$ & $42[27-90.5]$ \\
\hline
\end{tabular}

The median time to last seizure, in other words, the time it took for the medical professionals to stop the seizures was 5 days for the epilepsy and symptomatic group, but 10 days for the NORSE group. In-hospital mortality for SRSE (all causes) was 30\% (20), 15\% (2) for NORSE, 40\% (4) for pre-existing epilepsy and 32\% (14) for symptomatic. The median length of stay at OHSU (days) was 14.5 for epilepsy patients, 19.5 for symptomatic and 42 for NORSE patients. 
2. Comparisons of mortality between NORSE and pre-existing epilepsy patients with SRSE

To further assess the results, a Fisher's Exact test was completed between NORSE and pre-existing epilepsy patients. Table 2 summarizes the results of the Fisher Exact test of mortality and gender prevalence. The mortality for the NORSE group was $15 \%$ (2) and $40 \%$ (4) for preexisting epilepsy with an odds ratio of 3.453 and a p-value of 0.3413 . The female gender prevalence for the NORSE group was 69\% (9) and 40\% (4) for pre-existing epilepsy with an odds ratio of 3.189 and a p-value of 0.2215 (Table 2).

Table 2: Fisher Exact test results in regards to patient disposition and gender of NORSE and pre-existing epilepsy patients.

\begin{tabular}{|c|c|c|}
\hline & $\begin{array}{c}\text { NORSE } \\
(\mathbf{N}=\mathbf{1 3})\end{array}$ & $\begin{array}{c}\text { Pre-Existing Epilepsy } \\
\mathbf{( N = 1 0 )}\end{array}$ \\
\hline Alive & $11(85 \%)$ & $6(60 \%)$ \\
\hline Deceased & $2(15 \%)$ & $4(40 \%)$ \\
\hline $\begin{array}{c}\text { Odds Ratio for } \\
\text { Mortality }\end{array}$ & 3.453 & $\mathrm{p}=0.3413$ \\
\hline Male & $4(31 \%)$ & $6(60 \%)$ \\
\hline Female & $9(69 \%)$ & $4(40 \%)$ \\
\hline $\begin{array}{c}\text { Odds Ratio for } \\
\text { Gender }\end{array}$ & 3.189 & $\mathrm{p}=0.2215$ \\
\hline
\end{tabular}

Table 3 and 4 summarizes the Welch 2-sample t-test results for age of onset and the total length of stay for both NORSE and pre-existing epilepsy groups. For the age of onset, NORSE resulted in $45.4 \pm 14.1$ years, as opposed to epilepsy with $58.1 \pm 22.6$ years with a t-test value of 1.55 and a p-value of 0.1428 . Whereas for the total length of stay, NORSE resulted to $70.2 \pm 43.2$ years, while epilepsy resulted to $20.7 \pm 11.2$ years with t-test value of 3.96 and a p-value of 0.001412 . A Kaplan-Meier survival analysis was completed to compare NORSE and epilepsy patients, as shown below in Figure 2. 
Table 3: Welch 2-sample t-test results in regards to the age of onset of both NORSE and preexisting epilepsy as well as the resulting $t$ and $p$ values.

\begin{tabular}{|c|c|}
\hline Pre-Existing Epilepsy (N=10) & $58.1 \pm 22.6$ years \\
\hline NORSE (N=13) & $45.4 \pm 14.1$ years \\
\hline T & 1.55 \\
\hline $\mathbf{P}$ & 0.1428 \\
\hline
\end{tabular}

Table 4: Welch 2-sample t-test results in regards to the total length of stay at both OHSU and an outside hospital of both NORSE and pre-existing epilepsy as well as the resulting $t$ and $p$ values.

\begin{tabular}{|c|c|}
\hline $\begin{array}{c}\text { Pre-Existing Epilepsy } \\
(\mathbf{N}=\mathbf{1 0})\end{array}$ & $20.7 \pm 11.2$ years \\
\hline NORSE $\mathbf{( = 1 3 )}$ & $70.2 \pm 43.2$ years \\
\hline $\mathbf{T}$ & 3.96 \\
\hline $\mathbf{P}$ & 0.001412 \\
\hline
\end{tabular}

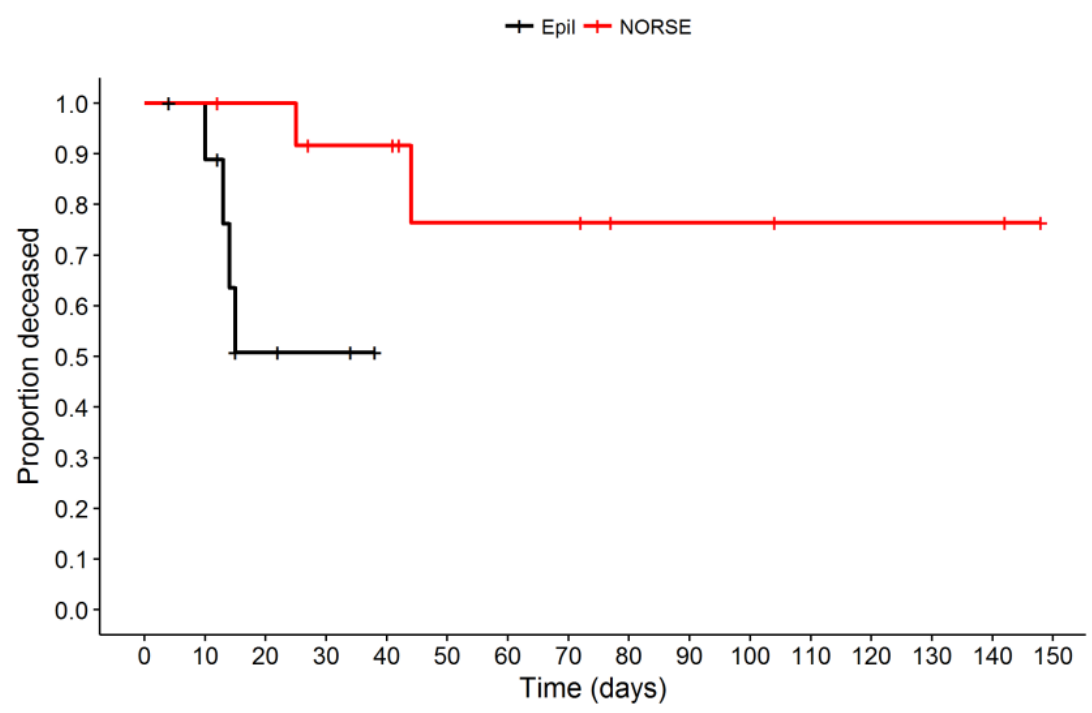

Figure 2: The Kaplan-Meier survival analysis in regards to the total length of stay (time in days) with $\beta=-2.272 \pm 1.135, z=-2.002$ and $p=0.0453$. The hazard ratio for NORSE was calculated to be 0.1031 . 
To examine the antibodies associated with NORSE, the NORSE patients were further categorized into four groups by antibody as follows: 1) positive for thyroid-related antibodies only, 2) positive for non-thyroid antibodies only, 3) positive for both thyroid and non-thyroid, and 4) negative antibody testing. Table 5 summarizes the results of the antibody testing in the NORSE patients. It is important to note that antibody testing was not completed on 1 NORSE patient, therefore one NORSE patient was excluded from Table 5 analysis. The group was organized into 4 categories consisting of: thyroid-related antibodies (TPO or Tg), non-thyroid related antibodies (neuronal VGKC), positive for both thyroid (TPO) and non-thyroid related antibodies (neuronal AChR) and negative antibody testing (often performed twice at separate testing centers). The four-potential possibility of antibody results group were then compared to data such as median onset age, female gender prevalence, median EEG length, median time to last seizure, median length of stay at OHSU, median number of steroids, CSF abnormality, ECT, ketogenic diet, the median number of immunotherapies and in-hospital mortality.

Table 5: Clinical characteristics of NORSE patients categorized by the results of antibody tests. One NORSE patient was excluded from this analysis because of not being antibody tested.

\begin{tabular}{|c|c|c|c|c|c|}
\hline \multicolumn{2}{|c|}{$\begin{array}{c}\text { Nhyroid Related } \\
\text { NORSE [13] }\end{array}$} & $\begin{array}{c}\text { Non-Thyroid } \\
(\mathbf{1})\end{array}$ & $\begin{array}{c}\text { Positive for } \\
\text { Both (1) }\end{array}$ & Negative (6) & Overall [12] \\
\hline Median Age & 50.5 & 34 & 68 & 42 & 46.25 \\
\hline Female Gender & $75 \%[3]$ & $100 \%[1]$ & $100 \%[1]$ & $50 \%[3]$ & $66.7 \%[8]$ \\
\hline Median EEG Length & 26 & 44 & 22 & 28.5 & 27.25 \\
\hline Median Time to Last Seizure & 12 & 2 & 10 & 9 & 10.5 \\
\hline Median Length of Stay at OHSU & 49.5 & 77 & 41 & 43 & 46.25 \\
\hline Median Number of Steroids & 2 & 2 & 1 & 1 & 1.5 \\
\hline CSF Abnormality & $100 \%[4]$ & $100 \%[1]$ & 0 & $83 \%[5]$ & $83.3 \%[10]$ \\
\hline ECT & $25 \%[1]$ & 0 & 0 & $50 \%[3]$ & $33.3 \%[4]$ \\
\hline Ketogenic Diet & $25 \%[1]$ & 0 & $100 \%[1]$ & 0 & $16.7 \%[2]$ \\
\hline Median number of & $2[1.5-2.5]$ & 3 & 2 & $0.5[0-1]$ & 2 \\
\hline Immunotherapies & $8 \%[1]$ & 0 & 0 & $8 \%[1]$ & $16.7 \%[2]$ \\
\hline In-hospital Mortality & & & & & \\
\hline
\end{tabular}




\section{Discussion}

The results of our study are consistent with prior reports: (1) NORSE is a rare condition that needs to be further studied, (2) NORSE subjects are more likely to be female, (3) there is a relationship with age, such that patients tend to be younger in the NORSE group compared to those with pre-existing epilepsy, (4) NORSE patients tend to be in the hospital for a more prolonged period of time compared to pre-existing epilepsy and symptomatic as well and (5) there are no antibodies clearly-associated with the condition.

Antibodies typically associated with NORSE are anti-NMDA receptor and anti-VGKC complex, although there are several cases with non-thyroid related (neuronal) antibodies as well. ${ }^{8}$ However, no specific antibody has been associated with NORSE. A novel finding in this study was that $50 \%$ of NORSE cases had positive antibody testing with majority (41.7\%) being thyroid-associated antibodies supporting the hypothesis that NORSE (or a subset of NORSE cases) may be autoimmune-mediated. As shown in Table 1, our results indicated that, as opposed to other etiologies, NORSE has a lower age of onset with a female gender prevalence and a prolonged in-hospital stay, refractory seizures and EEG duration. Compared to the other categories, NORSE did have a lower mortality rate, however that may be due to the rarity of NORSE, hence a lower reported incidence and a smaller sample size.

Pre-existing epilepsy and NORSE groups were further analyzed and compared to achieve a better analysis of the severity of NORSE due to the lower sample size (Table 2-4). An increased female gender prevalence was seen consistently in NORSE throughout the various data analysis. As shown in Table 2, 69\% (9) of NORSE patients were female, as opposed to 40\% (4) in the pre-existing epilepsy group. The odds ratio for gender is 3.189 with a p-value of 0.2215 , therefore it is not significant (Table 2). Although NORSE patients have a decreased mortality 
rate opposed to epilepsy, the odds ratio for mortality is 3.453 to 1 with a p-value of 0.3413 and is not significant. The difference between mortality and female gender prevalence is a difference that is not statistically significant, but that is said with high uncertainty due to a low number of subjects for the groups. NORSE patients tend to have a lower mean age of onset with a lower standard error $(\mathrm{t}=1.55$ and $\mathrm{p}=0.1428$ shown in Table 3$)$. Although the $\mathrm{p}$-value shows that the difference is statistically insignificant, the large measure of the variability in the results between epilepsy patients $(58.1 \pm 22.6$ years $)$ and NORSE patients $(45.4 \pm 14.1$ years $)$, reveals the imprecise results, which is more than likely due to the lower sample population. Table 4 revealed a low p-value of 0.001412 indicating that the difference is statistically significant, therefore, reiterating that the NORSE patients $(70.2 \pm 43.2$ years $)$ have a more prolonged length of stay as opposed to those with pre-existing epilepsy (20.7 \pm 11.2 years).

Based on the Kaplan-Meier curve and Cox regression analysis, the survival rate according to total length of stay resulted to a p-value of 0.0453 demonstrating the statistical significance between the NORSE and pre-existing epilepsy group. Since the coefficient represents the log change of hazard ratio of survival, it results to a hazard ratio of 0.1031 for NORSE. Therefore, the relative risk/risk ratio, with the assumption that the risk is constant over time, of in-hospital mortality is 9.699 times higher in those with pre-existing epilepsy. However, that may be due to the epilepsy group not having a prolonged hospital stay, therefore affecting the Kaplan-Meier survival curve analysis.

Based on Table 5, the in-hospital mortality for NORSE was $16.7 \%$ (2). In-hospital mortality would be $15 \%$ if we include the NORSE patient excluded from this analysis. Therefore, it is important to note that for Table 5 analysis, of the 13 NORSE cases, one subject was not antibody tested, therefore was excluded from this analysis due to no antibody testing 
results. The median age of onset was 46.25 and the majority of cases $(66.7 \%)$ were female. The average length of admission to OHSU was 46.25 days. Time on EEG-monitoring averaged 27.25 days, and time until seizure cessation was an average of 10.5 days.

Based on Table 5, four cases (33.3\%) had positive anti-thyroid related (TPO or Tg) antibodies. Of the 12 cases, only one (8.3\%) was positive for non-thyroid related (neuronal VGKC) anti-bodies and one (8.3\%) was positive for both thyroid-related (TPO) and non-thyroid related (neuronal AChR) antibodies. Therefore, a total of 5 (41.7\%) subjects had positive antithyroid related antibodies. The remaining six (50\%) cases had negative antibody testing (often performed twice at separate testing centers).

This study presents a novel aspect of understanding this rare fatal condition by establishing a potential autoimmune link that should be further studied. Previous studies have presented the hypothesis that NORSE (or a subset of NORSE cases) may be autoimmunemediated. However, there have been various antibody presence in NORSE with previous literature, but antibodies typically associated with NORSE are anti-NMDA receptor and antiVGKC complex, along with several cases presenting non-thyroid related (neuronal) antibodies as well. ${ }^{8}$ However, no specific antibody has been associated with NORSE. Based on a populationbased study at baseline of 5783 participants, $12.8 \%$ were positive for TPO antibodies with a higher prevalence in women compared to men. ${ }^{2}$ Therefore, the presence of $41.7 \%$ of NORSE cases with positive thyroid-associated antibodies not only supports the hypothesis that NORSE (or a subset of NORSE cases) may be autoimmune-mediated, but also further establishes a potential autoimmune link. It is important to note that those with positive thyroid related antibodies had a higher median age of onset, female gender prevalence, median time to last seizure, median length of stay at OHSU, median number of steroids, CSF abnormality, ketogenic 
diet, and median number of immunotherapies opposed to those with overall negative antibody testing. ECT was attempted on 33.3\% (4) of the 12 NORSE subjects antibody tested and it did not have any significant effect on improving the outcome, in fact, since ECT was attempted on patients in the groups with the 2 deaths, as shown in Table 5, it may have led to a decrease in the stability of the patient. Therefore, ECT may have worsened the condition of the patient. The results of this study, as shown in Table 1, also indicates that, as opposed to other etiologies, NORSE has a lower age of onset with a female gender prevalence and a prolonged in-hospital stay, refractory seizures and EEG duration. Compared to the other categories, NORSE did have a lower mortality rate, however that may be due to the rarity of NORSE hence a lower reported incidence.

There are numerous limitations in this study. The primary limitation is the small sample size, which is due to the rare nature of the condition. Another major limitation was incomplete data due to the retrospective nature of the study. Although all the data was taken from OHSU's database, there were several patients with very limited details on their chart due to several factors. For example, patients who have limited hospital visits and/or were transferred to OHSU tend to have limited data availability in regards to the patients' chart. Therefore, our results may be underestimating the true clinical characteristics and epidemiology of the patients. It is also important to note that since this was a retrospective chart review, the patients did not receive any form of systematic follow-up, which would have been very useful for this study. A significant limitation that has significantly affected the results presented in this study is the relatively small sample size, but that is due to NORSE being an extremely rare condition. In order to compensate for the limitation, we plan to initiate a multi-centered NORSE study. Establishing a binary system for mortality alters the data due to the severity of this condition resulting to poor 
outcomes. Since without follow-up data there is only one data point for each patient, the relatively small number of patients do not give sufficient data to understand patterns of mortality. While considering these limitations, the results established in this study are consistent with previous studies highlighting the clinical characteristics and epidemiology of NORSE: the condition is rare, patients tend to be younger, female-predominate, prolonged stay at the hospital, and there have been no antibodies clearly-associated with the condition.

Although a number of the results obtained in this study supported previous studies, our results failed to establish a statistical significance, which may be the result of a small sample size. Collectively, the results of this study highlight the importance and need for further research in NORSE to establish the clinical characteristics, epidemiology, and factors associated with NORSE in order to decrease the mortality and length of hospital stay, and therefore in essence, increase the possibility of a better outcome at disposition. 


\section{Conclusions:}

The results of this study are consistent with prior reports of NORSE epidemiology: the condition is rare, patients tend to be younger, female-predominate, and there have been no antibodies clearly-associated with the condition. However, 50\% of NORSE cases had positive antibody testing - the majority of which were thyroid-associated antibodies $(41.7 \%)$. While thyroid-associated antibodies are thought to be non-specific markers of inflammation and/or autoimmunity, the presence of $41.7 \%$ of NORSE cases with positive thyroid-associated antibodies not only supports the hypothesis that NORSE (or a subset of NORSE cases) may be autoimmune-mediated, but also further establishes a potential autoimmune link. Since NORSE is a rare, recently described life threatening syndrome of unclear etiology, more studies are needed to establish the epidemiology and mortality of NORSE to elucidate the underlying cause and optimal treatment. 


\section{References}

1. Wilder-Smith, E. P. V. et al. The NORSE (new-onset refractory status epilepticus) syndrome: defining a disease entity. Ann. Acad. Med. Singap. 34, 417-420 (2005).

2. Amouzegar, A. et al. The Prevalence, Incidence and Natural Course of Positive Antithyroperoxidase Antibodies in a Population-Based Study: Tehran Thyroid Study. PLoS One 12, (2017).

3. Spatola, M., Novy, J., Du Pasquier, R., Dalmau, J. \& Rossetti, A. O. Status epilepticus of inflammatory etiology. Neurology 85, 464-470 (2015).

4. Rossetti, A. O. \& Lowenstein, D. H. Management of refractory status epilepticus in adults. Lancet Neurol 10, 922-930 (2011).

5. Hirsch, L. J. et al. Proposed consensus definitions for new-onset refractory status epilepticus (NORSE), febrile infection-related epilepsy syndrome (FIRES), and related conditions. Epilepsia 59, 739-744 (2018).

6. Gaspard Nicolas et al. New-onset refractory status epilepticus (NORSE) and febrile infection-related epilepsy syndrome (FIRES): State of the art and perspectives. Epilepsia 59, $745-752$ (2018).

7. Shivani Ghoshal, P. date: F. 1, 2017 \& Lawrence J. Hirsch. NORSE: Cryptogenic New-Onset Refractory Status Epilepticus. Available at: http://www.mdedge.com/neurologyreviews/epilepsyresourcecenter/article/130565/epilepsyseizures/norse-cryptogenic-new-onset. (Accessed: 26th November 2017)

8. Gaspard, N. et al. New-onset refractory status epilepticus. Neurology 85, 1604-1613 (2015).

9. Kantanen, A.-M. et al. Incidence and mortality of super-refractory status epilepticus in adults. Epilepsy \& Behavior 49, 131-134 (2015). 
10. Van Lierde, I., Van Paesschen, W., Dupont, P., Maes, A. \& Sciot, R. De novo cryptogenic refractory multifocal febrile status epilepticus in the young adult: a review of six cases. Acta Neurol Belg 103, 88-94 (2003).

11. Costello, D. J., Kilbride, R. D. \& Cole, A. J. Cryptogenic New Onset Refractory Status Epilepticus (NORSE) in adults-Infectious or not? J. Neurol. Sci. 277, 26-31 (2009).

12. Shin, J.-W. et al. Clinical characterization of unknown/cryptogenic status epilepticus suspected as encephalitis: A multicenter cohort study. Journal of Neuroimmunology 315, 1-8 (2018).

13. Khawaja, A. M., DeWolfe, J. L., Miller, D. W. \& Szaflarski, J. P. New-onset refractory status epilepticus (NORSE) — The potential role for immunotherapy. Epilepsy \& Behavior 47, 17$23(2015)$.

14. Shorvon, S. \& Ferlisi, M. The treatment of super-refractory status epilepticus: a critical review of available therapies and a clinical treatment protocol. Brain 134, 2802-2818 (2011).

15. Kramer, U. et al. Febrile infection-related epilepsy syndrome (FIRES): pathogenesis, treatment, and outcome: a multicenter study on 77 children. Epilepsia 52, 1956-1965 (2011).

16. Baalen, A. van, Vezzani, A., Häusler, M. \& Kluger, G. Febrile Infection-Related Epilepsy Syndrome: Clinical Review and Hypotheses of Epileptogenesis. Neuropediatrics 48, 005018 (2017).

17. News, O. Fast facts. OHSU News Available at: https://news.ohsu.edu/news-media/facts. (Accessed: 26th May 2019)

18. Beghi, E. et al. Recommendation for a definition of acute symptomatic seizure. Epilepsia 51, $671-675$ (2010). 